\title{
Exenteración pélvica para cáncer de recto localmente avanzado y recurrente: resultados a largo plazo y factores pronósticos
}

\author{
Pelvic exenteration for locally advanced and recurrent rectal cancer: long-term outcomes \\ and prognostic factors
}

\author{
Omar Vergara-Fernández ${ }^{*}$, Francisco Armillas-Canseco", Carlos Sanjuán-Sánchez, \\ Emilio Sánchez-García-Ramos ${ }^{1}$ y Heriberto Medina-Franco ${ }^{2}$ \\ ${ }^{1}$ Departamento de Cirugía de Colon y Recto; ${ }^{2}$ Departamento de Cirugía Oncológica. Instituto Nacional de Ciencias Médicas y Nutrición Salvador \\ Zubirán, Ciudad de México, México
}

\begin{abstract}
Resumen
Objetivo: El objetivo de este trabajo fue analizar los resultados perioperatorios y a largo plazo de los pacientes sometidos a exenteración pélvica para cáncer de recto en un centro de referencia en la Ciudad de México. Método: Se incluyeron todos los pacientes que se sometieron a exenteración pélvica por cáncer de recto entre 1995 y 2019. Se analizaron variables demográficas, clínicas, quirúrgicas y patológicas. Resultados: Se incluyeron 18 pacientes operados por cáncer de recto (16 localmente avanzados y 2 recurrentes). La relación hombre: mujer fue de 1:3.5. La morbilidad mayor fue del 27.7\%. El sangrado intraoperatorio $\geq 1000 \mathrm{ml}$ se asoció con morbilidad (80 vs. $20 \%$; $p=0,029$ ) y mortalidad posoperatoria (100 vs. 0 ; $p=0.043)$. La mediana de sobrevida global fue 102 meses. Las sobrevidas global y libre de enfermedad a los 5 años fueron del $44.4 \%$ y el $38.8 \%$, respectivamente. La invasión linfovascular fue un factor de mal pronóstico para sobrevida libre de enfermedad $(p=0.017$ ). Conclusiones: La exenteración pélvica para el cáncer de recto es un procedimiento quirúrgico con altas morbilidad y mortalidad. La invasión linfovascular es un factor de mal pronóstico para la sobrevida libre de enfermedad.
\end{abstract}

Palabras clave: Exenteración pélvica. Cáncer de recto. Invasión linfovascular. CR-POSSUM.

\begin{abstract}
Introduction: Pelvic exenteration is a radical treatment for locally advanced and recurrent pelvic tumors. The aim of this study was to analyze the perioperative and long-term outcomes of patients undergoing pelvic exenteration for rectal cancer at a referral center in Mexico City. Method: We included all patients who underwent pelvic exenteration due to rectal cancer between 1995 and 2019. Demographic, clinical, surgical and pathological variables were analyzed. Results: 18 patients were included (16 locally advanced and 2 recurrent). The male-female ratio was 1:3.5. The highest morbidity was $27.7 \%$. Intraoperative bleeding $\geq 1000 \mathrm{ml}$ was associated with postoperative morbidity (80 vs. $20 \% ; p=0.029$ ) and mortality $(100 \mathrm{vs.} 0 ; p=0.043)$. The median overall survival was 102 months. Overall survival and disease free survival at 5 years after exenteration were $44.4 \%$ and $38.8 \%$, respectively. Lymphovascular invasion of the tumor was a poor prognostic factor for disease free survival
\end{abstract}

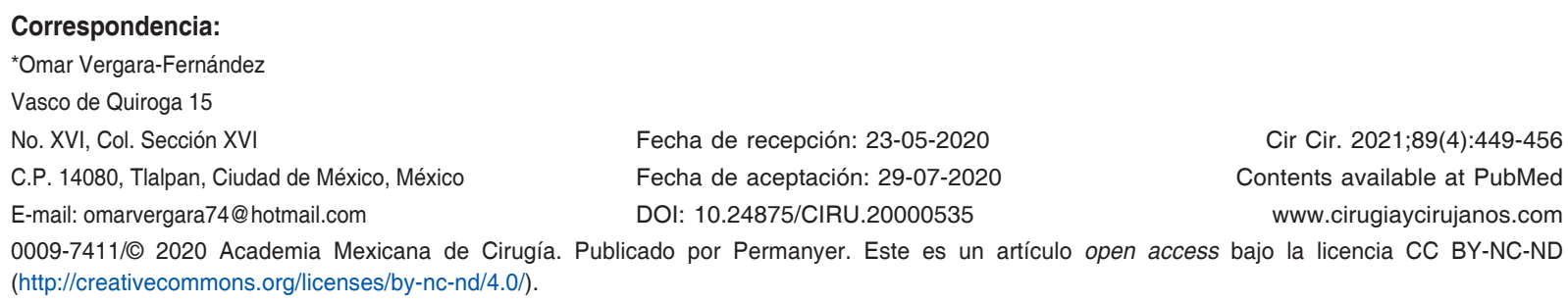

Fecha de recepción: 23-05-2020

Fecha de aceptación: 29-07-2020

DOI: 10.24875/CIRU.20000535

Cir Cir. 2021;89(4):449-456

Contents available at PubMed www.cirugiaycirujanos.com 
$(p=0.017)$. Conclusions: Pelvic exenteration for rectal cancer is a surgical procedure with high morbidity and mortality. Lymphovascular invasion is a poor prognostic factor for disease-free survival.

Key words: Pelvic exenteration. Rectal cancer. Lymphovascular invasion. CR-POSSUM.

\section{Introducción}

La exenteración pélvica es un procedimiento quirúrgico radical para las neoplasias pélvicas locales y recurrentes. Descrito por primera vez por Brunschwig en 1948, se diseñó inicialmente como un procedimiento paliativo para el tratamiento de tumores recurrentes de la vejiga y otros cánceres ginecológicos ${ }^{2,3}$, pero en la actualidad se considera como un potencial tratamiento curativo para el cáncer de recto localmente avanzado. En torno al 10\% de los cánceres colorrectales primarios presentan invasión tumoral en órganos adyacentes ${ }^{4}$. Por lo tanto, se puede ofrecer una resección multiorgánica para lograr unos márgenes quirúrgicos negativos, que es el factor más importante para la supervivencia a largo plazo ${ }^{5}$.

Clásicamente, la exenteración pélvica total se define como la extirpación del recto, el colon sigmoide, los uréteres distales, la vejiga, los órganos reproductivos internos, los ganglios linfáticos circundantes y el peritoneo pélvico. Además, puede ser necesaria la resección de músculos adyacentes, ligamentos y estructuras neurovasculares $u$ óseas.

El reconocimiento temprano de las recidivas observadas con la radioterapia y la atención multidisciplinaria ha aumentado la calidad de vida y ha reducido la necesidad de exenteración pélvica total ${ }^{6}$.

Sin embargo, si la estrategia terapéutica incluye exenteración pélvica, la morbilidad y la mortalidad deben considerarse y los pacientes tienen que ser seleccionados adecuadamente ${ }^{7}$. Además, los resultados posoperatorios dependen de la experiencia del equipo médico ${ }^{8}$.

En los últimos 60 años, los importantes avances médicos han hecho que la exenteración pélvica y otros procedimientos para el cáncer de recto sean mucho más seguros, lo que se demuestra por la tendencia a una menor tasa de mortalidad $^{9}$. El objetivo de este estudio es comparar los resultados perioperatorios y a largo plazo de la exenteración pélvica para el cáncer de recto en un centro de referencia en la Ciudad de México.

\section{Método}

Se realizó un análisis retrospectivo de todos los pacientes que se sometieron a una exenteración pélvica por cáncer de recto en un centro de referencia en la Ciudad de México entre 1995 y 2019. El diagnóstico de cáncer de recto se realizó mediante examen histopatológico. Se excluyeron los pacientes con registros o expedientes incompletos. Se recolectaron variables demográficas, clínicas, histopatológicas y quirúrgicas. Todos los pacientes se sometieron a un extenso estudio de estadificación preoperatoria, que incluía tomografía computarizada y resonancia magnética, de acuerdo con las pautas actuales.

Para cuantificar el número de compartimentos pélvicos resecados utilizamos la clasificación descrita por el Royal Marsden Hospital ${ }^{10}$. Todos los pacientes se clasificaron según la guía de la National Comprehensive Cancer Network (NCCN) versión 3.2019 para el cáncer de recto ${ }^{11}$. La mortalidad operatoria se definió la que ocurre en los primeros 90 días posteriores a la cirugía. La morbilidad se calificó de acuerdo con la clasificación de Clavien-Dindo, siendo la morbilidad mayor Clavien-Dindo III-V'2.

Se analizó la utilidad de la puntuación CR-POSSUM (Physiological and Operative Severity Score for the enUmeration of Mortality and Morbidity) ${ }^{13}$ como factor predictivo de mortalidad. El valor de corte fue de 10 puntos.

Utilizamos la razón de mortalidad esperada-observada (OEMR) para predecir la mortalidad del paciente (mortalidad observada/mortalidad calculada). También utilizamos el índice de comorbilidad de Charlson $^{14} \geq 3$ como factor de riesgo de morbimortalidad. Patólogos certificados por el instituto realizaron las evaluaciones histopatológicas. La recurrencia se diagnosticó mediante biopsia directa o estudio de imagen. La recurrencia local se definió como una recurrencia de la enfermedad dentro de la cavidad pélvica, y la recurrencia a distancia se definió como una recurrencia fuera de la cavidad pélvica. La invasión linfovascular se definió como la identificación de células tumorales que se propagan a áreas cubiertas por endotelio. El Comité de Revisión de Ética Institucional aprobó el estudio (SCI-1869-16/16-1).

El análisis descriptivo de las variables se basó en el tipo de variable. Las variables continuas se analizaron mediante una prueba no paramétrica, y se utilizó la prueba de ji al cuadrado para el contraste 
estadístico de las variables categóricas. Las tasas acumulativas y la influencia de los factores en la supervivencia se midieron utilizando el método de Kaplan-Meier, utilizando la prueba de log-rank. También se utilizó un modelo de riesgo proporcional de Cox para analizar los factores asociados con la sobrevida general y libre de enfermedad. Se consideró significativa una $p<0.05$.

No se crearon modelos para el análisis multivariado para evaluar variables independientes, ya que el grupo de estudio era demasiado pequeño para los parámetros identificados por el análisis univariado. El análisis estadístico se realizó con el programa SPSS versión 21.0 (SPSS, Chicago, IL, USA) para Windows.

\section{Resultados}

Entre 1995 y 2019, 18 pacientes se sometieron a exenteración pélvica por cáncer de recto primario y recurrente. Ocho (44.4\%) pacientes fueron intervenidos antes del año 2010. La relación hombre: mujer fue de 1:3.5. La edad mediana fue de 60 años (rango: 43-76). La mediana del índice de masa corporal fue de $24,15 \mathrm{~kg} / \mathrm{m}^{2}$ (rango: 16-48). La indicación quirúrgica fue cáncer rectal localmente avanzado en 16 pacientes $(88,8 \%)$ y cáncer rectal recurrente en $2(11,2 \%)$. En los pacientes operados por cáncer recurrente, este se identificó en una media de tiempo de 16 meses después de la cirugía de resección del tumor primario. Las características de los pacientes y las variables perioperatorias se describen en la tabla 1.

El procedimiento quirúrgico fue exenteración pélvica posterior en $15(83.3 \%)$ pacientes y exenteración pélvica anterior en 3 (16.6\%). El número de compartimentos pélvicos resecados fue similar en todos los procedimientos, ya que la mayoría de los pacientes tuvieron resección de cuatro o más compartimentos según la clasificación del Royal Marsden Hospital. La reconstrucción perineal con músculo recto abdominal transverso solo se realizó en tres casos (16,6\%). En $16(88.8 \%)$ pacientes se logró una resección R0.

Doce $(75 \%)$ pacientes recibieron algún tipo de terapia neoadyuvante y $8(44,4 \%)$ recibieron terapia adyuvante. Según el grado de diferenciación, 14 (77.7\%) casos fueron adenocarcinoma del recto moderadamente diferenciados, $2(11.1 \%)$ fueron bien diferenciados y otros $2(11.1 \%)$ fueron mal diferenciados.

La tasa global de complicaciones fue del $61,1 \%$, con una morbilidad mayor del 27,7\% (Clavien-Dindo III-IV).
Tabla 1. Características clínicas de los pacientes. Las variables estan presentadas en medianas (rango) o número (\%)

\begin{tabular}{|c|c|}
\hline General & $N(\%)$ \\
\hline Edad, años & $60(43-76)$ \\
\hline Sexo femenino & $14(77.8)$ \\
\hline Charlson $\geq 3$ & $6(33.3)$ \\
\hline$A S A \geq 3$ & $10(55.5)$ \\
\hline IMC & $24.15(16-31)$ \\
\hline Neoadyuvancia & $12(75)$ \\
\hline Sangrado transoperatorio (ml) & $700(200-6500)$ \\
\hline Duración de la cirugía (min) & $415(264-600)$ \\
\hline \multicolumn{2}{|l|}{ Compartimentos resecados } \\
\hline 2 & $2(11.1)$ \\
\hline 3 & $4(22.2)$ \\
\hline 4 & $8(44.4)$ \\
\hline 5 & $4(22.2)$ \\
\hline Construcción de colgajo & $3(16.6)$ \\
\hline Márgenes quirúrgicos negativos & $16(88.8)$ \\
\hline \multicolumn{2}{|l|}{ Estadio } \\
\hline l & $2(11.1)$ \\
\hline$\|$ & $9(50)$ \\
\hline III & $4(22.2)$ \\
\hline IV & $3(16.7)$ \\
\hline Complicación posoperatoria & $11(61.1)$ \\
\hline \multicolumn{2}{|l|}{ Clavien-Dindo } \\
\hline I & 4 \\
\hline II & 2 \\
\hline III & 1 \\
\hline IV & 2 \\
\hline V & 2 \\
\hline Estancia hospitalaria (días) & $10(7-42)$ \\
\hline Terapia adyuvante & $8(44.4)$ \\
\hline \multicolumn{2}{|l|}{ Fecha de la cirugía } \\
\hline Después de 2010 & $10(55.5)$ \\
\hline Hasta 2010 & $8(44.4)$ \\
\hline
\end{tabular}

Tabla 2. Complicaciones tras la exenteración pélvica

\begin{tabular}{ll}
\hline General & N (\%) \\
\hline Colección pélvica o absceso & $4(22.2)$ \\
Dehiscencia de la herida & $2(11.1)$ \\
Íleo u obstrucción del intestino delgado & $3(16.6)$ \\
Hemorragia posoperatoria tardía & $3(16.6)$ \\
Lesión o infección del tracto urinario & $1(0.5)$ \\
Neumonía & $2(11.1)$ \\
Otras (insuficiencia renal aguda, infarto agudo de & $2(11.1)$ \\
miocardio) & \\
\hline
\end{tabular}


Estas complicaciones fueron más frecuentes en aquellos procedimientos realizados antes del año 2010 (40 vs 60\%). La mayoría de las complicaciones se manejaron de forma conservadora. Solo 3 (16.6\%) pacientes requirieron una intervención quirúrgica que incluyó drenaje de absceso/colección abdominal. El perfil de las complicaciones posoperatorias se describe en la tabla 2. Encontramos una tasa de morbilidad mayor más alta en los pacientes con una puntuación ASA (American Society of Anesthesiologists) $\geq 3$ (100 vs. $0 \% ; p=0.047$ ). El sangrado intraoperatorio $\geq 1000 \mathrm{ml}$ también se relacionó significativamente con la morbilidad mayor ( 80 vs. $20 \% ; p=0.029$ ) (Tabla 3).

Tres (16.6\%) pacientes murieron dentro de los 90 días posteriores a la cirugía. Las causas fueron sepsis abdominal con insuficiencia orgánica múltiple en un paciente y neumonía intrahospitalaria en los otros dos. No hubo relación entre la mortalidad posoperatoria y el año en que se realizó el procedimiento. Notamos que los pacientes con un sangrado intraoperatorio $\geq 1000 \mathrm{ml}$ también estaban relacionados con mayor mortalidad posoperatoria ( $100 \mathrm{vs.} 0 \% ; p=0.043$ ) (Tabla 3).

Con respecto a la puntuación CR-POSSUM, $5(27.7 \%)$ pacientes tuvieron $>10$ puntos. La mortalidad pronosticada por CR-POSSUM y la mortalidad observada fueron del $9.6 \%$ y el $16.6 \%$, respectivamente $(p=0.059)$. En la tabla 3 se detalla el análisis univariado de los factores asociados con la morbilidad y la mortalidad posoperatorias.

\section{Seguimiento y sobrevida}

Durante una mediana de seguimiento de 38 meses (rango: 10-66), 4 (22.2\%) pacientes murieron, uno debido a la progresión del cáncer y tres por razones no oncológicas (neumonía, leucemia y enfermedad cerebrovascular).

La mediana de sobrevida global para toda la cohorte fue de 102 meses (rango: 3-166). Esta mediana de sobrevida global fue menor para aquellos pacientes operados antes del año 2010 (51.2 vs. 76.9 meses). La tasa de recurrencia global fue del $33.3 \%$, con un tiempo de recurrencia promedio de 24.5 meses (rango: $5-54$ ), con un $40 \%$ de recidivas locorregionales y un $60 \%$ de recidivas distantes. Solo un paciente tuvo recurrencia local y distante. Al final de este estudio, cuatro pacientes tenían enfermedad metastásica (tres en el hígado y uno en la glándula suprarrenal). La sobrevida general (SG) y la sobrevida libre de
Tabla 3. Análisis univariado de factores asociados a morbilidad mayor y a mortalidad posoperatorias (90 días)

\begin{tabular}{|c|c|c|c|c|c|}
\hline \multirow[b]{2}{*}{ Variables } & \multicolumn{2}{|c|}{ Morbilidad } & \multicolumn{3}{|c|}{ Mortalidad } \\
\hline & $\begin{array}{c}\text { Sí } \\
N=5 \\
(\%)\end{array}$ & $\begin{array}{c}\text { No } \\
N=13 \\
(\%)\end{array}$ & $\begin{array}{c}\text { Sí } \\
N=3 \\
(\%)\end{array}$ & $\begin{array}{c}\text { No } \\
N=15 \\
(\%)\end{array}$ & \\
\hline Edad & \multicolumn{3}{|c|}{0.500} & \multirow{2}{*}{\multicolumn{2}{|c|}{$\begin{array}{l}7(46.6) \\
8(53.4)\end{array}$}} \\
\hline $\begin{array}{l}\geq 60 \text { años } \\
<60 \text { años }\end{array}$ & $\begin{array}{l}3(60) \\
2(40)\end{array}$ & $\begin{array}{l}6(46.2) \\
7(53.8)\end{array}$ & $\begin{array}{l}2(66.6) \\
1(33.3)\end{array}$ & & \\
\hline Sexo & \multicolumn{3}{|c|}{0.533} & \multirow{3}{*}{\multicolumn{2}{|c|}{$\begin{array}{l}2(13.4)^{0.108} \\
13(86.6)\end{array}$}} \\
\hline Femenino & $3(60)$ & $11(84.6)$ & $1(33.3)$ & & \\
\hline Masculino & $2(40)$ & $2(15.4)$ & $2(66.6)$ & & \\
\hline Charlson & \multicolumn{3}{|c|}{0.385} & \multirow{3}{*}{\multicolumn{2}{|c|}{$\begin{array}{l}5(33.4) \\
10(66.6)\end{array}$}} \\
\hline$\geq 3$ & $2(40)$ & $4(30.8)$ & $1(33.3)$ & & \\
\hline$<3$ & $3(60)$ & $9(69.2)$ & $2(66.6)$ & & \\
\hline ASA & \multicolumn{3}{|c|}{0.036} & \multirow{3}{*}{\multicolumn{2}{|c|}{$\begin{array}{l}6(40)^{0.216} \\
9(60)\end{array}$}} \\
\hline$\geq 3$ & $5(100)$ & $5(38.5)$ & $3(100)$ & & \\
\hline$<3$ & 0 & $8(61.5)$ & 0 & & \\
\hline IMC & \multicolumn{3}{|c|}{0.314} & \multirow{3}{*}{\multicolumn{2}{|c|}{$\begin{array}{l}0.216 \\
8(53.4) \\
7(46.6)\end{array}$}} \\
\hline$\geq 25$ & $1(20)$ & $7(53.8)$ & 0 & & \\
\hline$<25$ & $4(80)$ & $6(46.15)$ & $3(100)$ & & \\
\hline $\begin{array}{l}\text { Sangrado } \\
\text { transoperatorio (ml) }\end{array}$ & \multicolumn{3}{|c|}{0.047} & \multirow{3}{*}{\multicolumn{2}{|c|}{$\begin{array}{c}4(26.6) \\
11(73.4)\end{array}$}} \\
\hline$\geq 1000$ & $4(80)$ & $3(23.1)$ & $3(100)$ & & \\
\hline$<1000$ & $1(20)$ & $10(76.9)$ & 0 & & \\
\hline $\begin{array}{l}\text { Duración de la } \\
\text { cirugía (min) }\end{array}$ & \multicolumn{3}{|c|}{0.701} & \multirow{3}{*}{\multicolumn{2}{|c|}{$\begin{array}{l}10(66.6) \\
5(33.4)\end{array}$}} \\
\hline$\geq 360$ & $5(100)$ & $8(61.5)$ & $3(100)$ & & \\
\hline$<360$ & 0 & $5(38.5)$ & 0 & & \\
\hline $\begin{array}{l}\text { Compartimentos } \\
\text { resecados }\end{array}$ & \multicolumn{3}{|c|}{0.561} & \multicolumn{2}{|c|}{$\begin{array}{c}10(66.6) \\
5(33.4)\end{array}$} \\
\hline$>3$ & $4(80)$ & $9(69.2)$ & $2(66.6)$ & & \\
\hline$<3$ & $1(20)$ & $4(30.8)$ & $1(33.3)$ & & \\
\hline CR-POSSUM & \multicolumn{3}{|c|}{0.608} & \multirow{3}{*}{\multicolumn{2}{|c|}{$\begin{array}{l}5(33.4) \\
10(66.6)\end{array}$}} \\
\hline$\geq 10$ & $3(60)$ & $5(38.5)$ & $01(33.3)$ & & \\
\hline$<10$ & $2(40)$ & $8(61.5)$ & $3(100)$ & & \\
\hline Fecha de la cirugía & \multicolumn{3}{|c|}{0.618} & \multirow{3}{*}{\multicolumn{2}{|c|}{$\begin{array}{ll} & 0.589 \\
9(60) & \\
6(40) & \end{array}$}} \\
\hline Después de 2010 & $2(40)$ & $8(61.5)$ & 1 (33.3) & & \\
\hline Hasta 2010 & $3(60)$ & $5(38.5)$ & $2(66.6)$ & & \\
\hline
\end{tabular}

enfermedad (SLE) a 5 años para toda la cohorte fueron del $44.4 \%$ y el $38.8 \%$, respectivamente (Fig. 1 ).

\section{Factores pronósticos para sobrevida global y sobrevida libre de enfermedad}

El análisis univariado demostró dos factores de mal pronóstico estadísticamente significativos para la supervivencia. La invasión linfovascular, que estuvo presente en $5(55.5 \%)$ pcientes, tuvo significancia estadística para la SLE $(p=0.017)$, y la invasión 

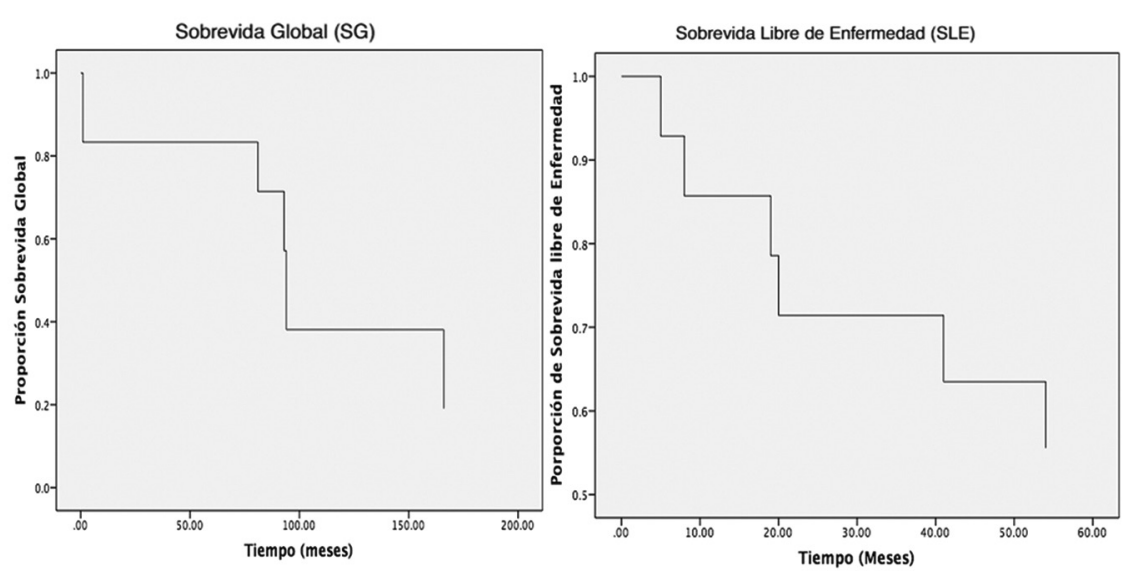

Figura 1. Curva de Kaplan-Meier de sobrevida global y sobrevida libre de enfermedad a 5 años para toda la cohorte.

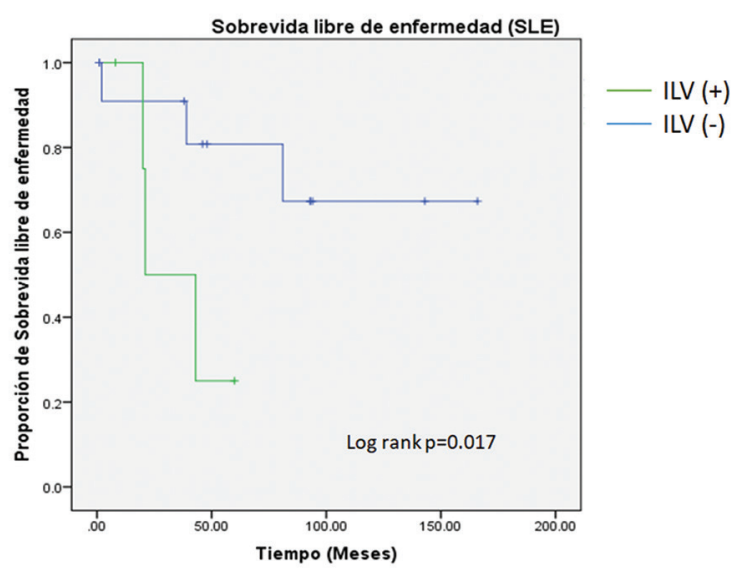

Figura 2. Curva de Kaplan-Meier de sobrevida libre de enfermedad según la presencia (línea verde) o la ausencia (línea azul) de invasión linfovascular del tumor. ILV: invasión linfovascular; SLE: sobrevida libre de enfermedad.

perineural, que estuvo presente en 4 (22.2\%) pacientes, mostró una tendencia hacia la significancia estadística $(p=0.064)$. Estos resultados no fueron consistentes para la SG (Fig. 2).

Por otro lado, encontramos que el sexo femenino se asoció con un mal pronóstico en la $S G(p=0.031)$. El estadio clínico, los márgenes quirúrgicos, los ganglios linfáticos, la enfermedad metastásica, el uso de terapia adyuvante o neoadyuvante, y el año en que se realizó el procedimiento, no tuvieron un impacto significativo en el pronóstico de sobrevida (Tabla 4).

\section{Discusión}

La exenteración pélvica se describió por primera vez como un procedimiento paliativo para neoplasias ginecológicas, y más adelante se describió para el cáncer rectal localmente avanzado y recurrente ${ }^{15,16}$. La mortalidad notificada en la primera serie fue del $30 \%$ y no hubo supervivientes a largo plazo', pero las publicaciones más recientes muestran que se ha reducido de 0 a $10 \%{ }^{17}$, lo que demuestra una mejora en los estándares de atención médica. La mortalidad perioperatoria reportada en esta serie es del 16.6\% (3 pacientes), lo que refleja que la mortalidad de la exenteración pélvica refleja un acercamiento a la de la resección radical electiva para el cáncer colorrectal primario ${ }^{18}$.

Aunque no es estadísticamente significativo, un valor > 10 en la puntuación CR-POSSUM mostró una tendencia estadística de mortalidad a los 30 días $(p=0.059)$. En este punto, ninguna otra serie ha utilizado la puntuación CR-POSSUM en la exenteración pélvica, pues generalmente se usa para la cirugía colorrectal electiva con o sin cáncer ${ }^{19,20}$. Cheung, et al. ${ }^{21}$ explican que CR-POSSUM se desarrolló para predecir la mortalidad posoperatoria a los 30 días en los pacientes programados para cirugía colorrectal, y que su incapacidad para predecir la supervivencia podría deberse a que no se diseñó para predecir la morbilidad a los 30 días y por tanto no puede establecerse el vínculo entre esta y la supervivencia a largo plazo. El uso de CR-POSSUM se ha recomendado como lo más adecuado para la práctica quirúrgica ${ }^{22}$.

La morbilidad posquirúrgica en la exenteración pélvica varía ampliamente, del $37 \%$ al $100 \%$ reportada por Yang et al. ${ }^{23}$. Nuestros resultados muestran complicaciones mayores en el $27.7 \%$ de los pacientes, incluyendo infecciones, hemorragia posoperatoria, eventos tromboembólicos y muerte. Estos resultados se comparan favorablemente con los informados por Nielsen, et 
Tabla 4. Análisis univariado de los factores asociados a la sobrevida general y la sobrevida libre de enfermedad a los 5 años

\begin{tabular}{|c|c|c|c|c|c|}
\hline & \multirow[t]{2}{*}{$\mathrm{N}(\%)$} & \multicolumn{2}{|c|}{ SG 5 años } & \multicolumn{2}{|c|}{ SLE 5 años } \\
\hline & & $\%$ & $\mathrm{p}$ & $\%$ & $p$ \\
\hline \multirow{3}{*}{$\begin{array}{l}\text { Edad } \\
\quad<60 \text { años } \\
>60 \text { años }\end{array}$} & & & \multirow[t]{3}{*}{0.905} & & \multirow[t]{3}{*}{0.179} \\
\hline & $9(50)$ & 44.4 & & 33.3 & \\
\hline & $9(50)$ & 44.4 & & 44.4 & \\
\hline \multicolumn{2}{|l|}{ Sexo } & & \multirow[t]{3}{*}{0.031} & & \multirow[t]{3}{*}{0.891} \\
\hline Masculino & 4 (22.2) & 25 & & 0 & \\
\hline Femenino & $14(77.7)$ & 25 & & 50 & \\
\hline \multicolumn{2}{|l|}{ Terapia neoadyuvante } & & \multirow[t]{3}{*}{0.557} & & \multirow[t]{3}{*}{0.282} \\
\hline Sí & $12(66.7)$ & 33.3 & & 25 & \\
\hline No & $6(33.3)$ & 66.6 & & 66.6 & \\
\hline \multicolumn{2}{|l|}{ Estado de márgenes } & & \multirow[t]{3}{*}{0.992} & & \multirow[t]{3}{*}{0.712} \\
\hline RO & $16(88.9)$ & 43.7 & & 37.5 & \\
\hline $\mathrm{R} 1$ & $2(11.1)$ & 50 & & 50 & \\
\hline \multicolumn{2}{|l|}{ ILV } & & \multirow[t]{3}{*}{0.830} & & \multirow[t]{3}{*}{0.017} \\
\hline Sí & $5(27.8)$ & 0 & & 0 & \\
\hline No & $13(72.2)$ & 61.5 & & 53.8 & \\
\hline \multicolumn{2}{|l|}{ PNI } & 25 & \multirow[t]{3}{*}{0.484} & & \multirow[t]{3}{*}{0.064} \\
\hline Sí & 4 (22.2) & 50 & & 25 & \\
\hline No & $14(77.8)$ & & & 42.8 & \\
\hline \multicolumn{2}{|l|}{ Ganglios linfáticos } & & \multirow[t]{3}{*}{0.641} & & \multirow[t]{3}{*}{0.402} \\
\hline No & $13(72.2)$ & 38.4 & & 30.7 & \\
\hline $\mathrm{N}+$ & $5(27.8)$ & 60 & & 60 & \\
\hline \multirow{4}{*}{$\begin{array}{l}\text { Enfermedad } \\
\text { metastásica } \\
\text { M0 } \\
\mathrm{M}_{+}\end{array}$} & & & \multirow[t]{4}{*}{0.454} & & \multirow[t]{4}{*}{0.67} \\
\hline & 15 (83.3) & 53.3 & & 46.6 & \\
\hline & $3(16.7)$ & 0 & & 0 & \\
\hline & & & & & \\
\hline \multicolumn{2}{|l|}{ Terapia adyuvante } & & 0.884 & & 0.202 \\
\hline Sí & $10(55.6)$ & 30 & & 20 & \\
\hline No & $8(44.5)$ & 62.5 & & 62.5 & \\
\hline CR-POSSUM & & & 0.095 & & 0.376 \\
\hline$>10$ & $8(44.5)$ & 37.5 & & 37.5 & \\
\hline$<10$ & $10(55.5)$ & 50 & & 40 & \\
\hline Fecha de cirugía & & & 0.461 & & 0.429 \\
\hline Después de 2010 & $10(55.6)$ & 70 & & 20 & \\
\hline Hasta 2010 & $8(44.5)$ & 50 & & 62.5 & \\
\hline
\end{tabular}

CR-POSSUM: Physiological and Operative Severity Score for the enUmeration of Mortality and Morbidity; LVI: invasión linfovascular; PNI: invasión perineural; SG: sobrevida general; SLE: sobrevida libre de enfermedad.

al. ${ }^{24}$ y por el grupo del Royal Marsden $(34,5-42,2 \%)^{25}$. Como encontramos en nuestra serie, la infección de la herida quirúrgica y el absceso/colección pélvica se mencionan como las complicaciones más frecuentes en otras series ${ }^{26}$. Nuestro análisis muestra que los pacientes con una puntuación ASA $\geq 3$ tuvieron mayor morbilidad ( $p=0.036$ ). Incluso después de la estandarización basada en el tipo de procedimiento se ha encontrado una fuerte asociación entre el estado físico ASA y la morbilidad posoperatoria para la cirugía colorrectal ${ }^{26}$.
El sangrado intraoperatorio $\geq 1000 \mathrm{ml}(p=0.043)$ fue una variable estadísticamente significativa para la mortalidad posoperatoria $(p=0.043)$, así como la morbilidad mayor $(p=0.047)$. Probablemente esto podría estar relacionado con el tipo de procedimiento y el número de compartimentos resecados, ya que seis de los siete casos que cumplieron con este parámetro tenían más de tres compartimentos pélvicos resecados según la clasificación del Royal Marsden Hospital26. Hsu, et al. ${ }^{27}$ señalan que la exenteración pélvica abarca un grupo de cirugías complejas que incluyen la resección de múltiples órganos. La eliminación de estos tejidos en la fascia endopélvica crea un gran espacio muerto que predispone a la formación de abscesos y explicaría la alta tasa de complicaciones. Por lo tanto, creemos que un procedimiento más complejo se asocia con una mayor mortalidad y más morbilidad.

A pesar de encontrar menores tasas de morbilidad en las cirugías realizadas después del año 2010, no hubo diferencias significativas en cuanto a morbilidad mayor según el año en que fueron realizados los procedimientos (60 vs. $40 \% ; p=0.618$ ). Varios factores han influido en los resultados en los últimos años, como la integración de antibióticos de amplio espectro, la profilaxis tromboembólica, los dispositivos de energía avanzados en cirugía, la experiencia quirúrgica y los equipos de cuidados críticos $^{28}$. Como resultado, se ha logrado una mayor supervivencia a largo plazo con una morbilidad aceptable en pacientes seleccionados en unidades especializadas.

La resección completa (R0) se logró en la mayoría de los pacientes, lo que se compara favorablemente con otras series publicadas ${ }^{29}$. Se sabe que los márgenes de resección negativos se asocian con una supervivencia significativamente mayor, pero la proporción de pacientes con resección R1-R2 es sustancialmente mayor en otras series ${ }^{30,31}$, por lo que en nuestra serie no puede establecerse esta asociación.

Varios autores informan de los resultados de la exenteración pélvica para el cáncer colorrectal con una amplia variación en términos de supervivencia, lo que refleja los tamaños de muestra limitados de algunos centros de estudio. En nuestra serie, la supervivencia a 5 años se compara de manera similar con la referida en la literatura, que es del $46-66 \% \%^{32-34}$. La SG y la SLE fueron del $44.4 \%$ y el $38.8 \%$, respectivamente. Katory, et al. ${ }^{35}$ han publicado una serie de 14 pacientes sometidos a una exenteración pélvica por cáncer colorrectal, alcanzando una SG a 5 años superior al $80 \%$. Estos hallazgos son concordantes con los de nuestra serie, encontrando una SG mayor en los pacientes operados 
después del año 2010, aunque la relación entre el año del procedimiento y la supervivencia no demostró ser significativa (70 vs. $50 \% ; p=461$ ).

Los estudios retrospectivos han reconocido factores pronósticos asociados con mejores resultados que concuerdan con los ya reconocidos para el cáncer de recto, incluyendo el estadio, la afectación de los ganglios linfáticos y los márgenes quirúrgicos positivos $^{34,36}$. Nuestra serie no identificó estas variables como estadísticamente significativas.

El análisis univariado mostró que el sexo femenino es un factor altamente significativo para la supervivencia $(p=0.03)$. En nuestro conocimiento, ninguna serie ha establecido el sexo femenino como un factor de mal pronóstico en la supervivencia. La invasión linfovascular fue el factor pronóstico identificado más importante, con significancia estadística para la SLE $(p=0.017)$. La invasión perineural también mostró una tendencia hacia la significancia estadística $(p=0.064)$. La invasión linfovascular ha demostrado ser un factor pronóstico independiente para la recurrencia y la mortalidad en la cirugía colorrectal ${ }^{36,37}$. Se cree que ambas variables denotan un fenotipo agresivo, una diferenciación pobre y una mayor capacidad invasiva, y por lo tanto son de impacto en la SLE en los pacientes con cáncer colorrectal ${ }^{38}$.

\section{Limitaciones}

Nuestro estudio tiene limitaciones, como su naturaleza retrospectiva, un tamaño de muestra pequeño y la inclusión heterogénea de pacientes con cáncer rectal primario y recurrente. Como mencionamos, ha habido cambios en la práctica durante el periodo de estudio, como la estadificación, la terapia neoadyuvante, las técnicas quirúrgicas, etc., y por ello esta serie puede no reflejar la práctica quirúrgica contemporánea para la exenteración pélvica. En ausencia de ensayos aleatorizados para cualquiera de estos resultados, los médicos deben confiar en los datos de estudios no controlados o de cohortes para este grupo de pacientes. Es importante continuar registrando los pacientes que se someten a una exenteración pélvica para determinar si la importancia estadística de los factores identificados en este estudio permanece constante.

\section{Conclusiones}

En conclusión, la exenteración pélvica es un procedimiento agresivo con unas considerables morbilidad y mortalidad, que son aceptables por el tipo de cirugía extensa y radical que representa. Es bien sabido que estos beneficios disminuyen en los casos en que no se puede alcanzar una resección R0, lo que aumenta el riesgo de complicaciones posoperatorias. Creemos que es importante utilizar puntajes predictivos, como CR-POSSUM, para seleccionar adecuadamente a los pacientes que pueden ser candidatos para la exenteración pélvica. En nuestra serie, la presencia de invasión linfovascular en la patología final se identificó como un factor de mal pronóstico para la SLE.

\section{Conflicto de intereses}

Los autores declaran que no existe ningún conflicto de intereses.

\section{Financiamiento}

No existió financiamiento para este estudio.

\section{Responsabilidades éticas}

Protección de sujetos humanos y animales. Los autores declaran que no se realizaron experimentos en humanos o animales para este estudio.

Confidencialidad de los datos. Los autores declaran que han seguido los protocolos de su centro de trabajo sobre la publicación de datos de pacientes.

Derecho a la privacidad y consentimiento informado. Los autores declaran que no aparecen datos de pacientes en este artículo.

\section{Bibliografía}

1. Brunschwig A. Complete excision of pelvic viscera for advanced carcinoma; a one-stage abdominoperineal operation with end colostomy and bilateral ureteral implantation into the colon above the colostomy. Cancer. 1948;1:177-83.

2. Lim RS, Yang TX, Chua TC. Postoperative bladder and sexual function in patients undergoing surgery for rectal cancer: a systematic review and meta-analysis of laparoscopic versus open resection of rectal cancer. Tech Coloproctol. 2014;18:993-1002.

3. Terán-Porcayo MA, Zeichner-Gancz I, del-Castillo RACG, Beltrán-Ortega A, Solorza-Luna G. Pelvic exenteration for recurrent or persistent cervical cancer: experience of five years at the National Cancer Institute in Mexico. Med Oncol. 2006;23:219-23.

4. Pawlik TM, Skibber JM, Rodríguez-Bigas MA. Pelvic exenteration for advanced pelvic malignancies. Ann Surg Oncol. 2006;13:612-23.

5. Wanebo HJ, Antoniuk P, Koness RJ, Levy A, Vezeridis M, Cohen SI, et al. Pelvic resection of recurrent rectal cancer: technical considerations and outcomes. Dis Colon Rectum. 1999;42:1438-48.

6. Moffat FL, Falk RE. Radical surgery for extensive rectal cancer: is it worthwhile? Berlin, Heidelberg: Springer; 1998. p. 71-83.

7. Rodríguez-Bigas MA, Petrelli NJ. Pelvic exenteration and its modifications. Am J Surg. 1996;171:293-301.

8. Yeung RS, Moffat FL, Falk RE. Pelvic exenteration for recurrent and extensive primary colorectal adenocarcinoma. Cancer. 1993;72:1853-8.

9. Vergara-Fernández O, Swallow CJ, Victor JC. Assessing outcomes following surgery for colorectal cancer using quality of care indicators. Can J Surg. 2010;53:232-40. 
10. Beyond TME Collaborative: Bhangu A, Beynon J, Brown G, Chang G, Das $P$, Desai $A$, et al. Consensus statement on the multidisciplinary management of patients with recurrent and primary rectal cancer beyond total mesorectal excision planes. Br J Surg. 2013;100:E1-E33.

11. NCCN Clinical Practice Guidelines in Oncology. Disponible en: https:// www.nccn.org/professionals/physician_gls

12. Dindo D, Demartines N, Clavien P-A. Classification of surgical complications: a new proposal with evaluation in a cohort of 6336 patients and results of a survey. Ann Surg. 2004;240:205-13.

13. Charlson ME, Pompei P, Ales KL, MacKenzie CR. A new method of classifying prognostic comorbidity in longitudinal studies: development and validation. J Chronic Dis. 1987:40:373-83.

14. Tekkis PP, Prytherch DR, Kocher HM, Senapati A, Poloniecki JD, Stamatakis JD, et al. Development of a dedicated risk-adjustment scoring system for colorectal surgery (colorectal POSSUM). $\mathrm{Br} \mathrm{J}$ Surg. 2004:91:1174-82.

15. Brintnall ES, Flocks RH. En masse pelvic viscerectomy with uretero-intestinal anastomosis. AMA Arch Surg. 1950;61:851-68.

16. Wanebo HJ, Marcove RC. Abdominal sacral resection of locally recurrent rectal cancer. Ann Surg. 1981;194:458-71.

17. Lopez MJ, Standiford SB, Skibba JL. Total pelvic exenteration. A 50-year experience at the Ellis Fischel Cancer Center. Arch Surg. 1994;129:390-5.

18. Heriot AG, Byrne CM, Lee P, Dobbs B, Tilney H, Solomon MJ, et al. Extended radical resection: the choice for locally recurrent rectal cancer Dis Colon Rectum. 2008:51:284-91.

19. Ramkumar $\mathrm{T}, \mathrm{Ng}$ V, Fowler L, Farouk R. A comparison of POSSUM, P-POSSUM and colorectal POSSUM for the prediction of postoperative mortality in patients undergoing colorectal resection. Dis Colon Rectum. 2006:49:330-5.

20. Horzic M, Kopljar M, Cupurdija K, Bielen DV, Vergles D, Lackovic Z. Comparison of P-POSSUM and Cr-POSSUM scores in patients undergoing colorectal cancer resection. Arch Surg. 2007:142:1043-8.

21. Cheung H, Poon JTC, Law W-L. The impact of POSSUM score on the long-term outcome of patients with rectal cancer. Colorectal Dis. 2013;15:1171-6.

22. Jones $\mathrm{HJ}$, de Cossart $\mathrm{L}$. Risk scoring in surgical patients. $\mathrm{Br} \mathrm{J}$ Surg. 1999;86:149-57.

23. Yang TX, Morris DL, Chua TC. Pelvic exenteration for rectal cancer: a systematic review. Dis Colon Rectum. 2013:54:519-31.

24. Nielsen MB, Rasmussen PC, Lindegaard JC, Laurberg S. A 10ロyear experience of total pelvic exenteration for primary advanced and locally recurrent rectal cancer based on a prospective database. Colorectal Dis. 2012:14:1076-83.
25. Banghu A, Ali SM, Nicholis RJ, Tekkis P. Indications and aoutcome of pelvic exenteration for locally advanced primary and recurrent rectal cancer. Ann Surg. 201;259:315-22.

26. Oh NH, Kim KJ. Outcomes and risk factors affecting mortality in patients who underwent colorectal emergency surgery. Ann Coloproctol. 2016:32:133-8.

27. Hsu T-W, Chiang F-F, Chen M-C, Wang H-M. Pelvic exenteration for men with locally advanced rectal cancer: a morbidity analysis of complicated cases. Asian J Surg. 2011;34:115-20.

28. Brown KGM, Solomon MJ, Koh CE. Pelvic exenteration surgery: the evolution of radical surgical techniques for advanced and recurrent pelvic malignancy. Dis Colon Rectum. 2017;60:745-54.

29. Rottoli M, Vallicelli C, Boschi L, Poggioli G. Outcomes of pelvic exenteration for recurrent and primary locally advanced rectal cancer. Int $\mathrm{J}$ Surg. 2017;48:69-73.

30. Ferenschild FTJ, Vermaas M, Verhoef C, Ansink AC, Kirkels WJ, Eggermont AMM, et al. Total pelvic exenteration for primary and recurrent malignancies. World J Surg. 2009;33:1502-8.

31. Yang HY, Park SC, Hyun JH, Seo HK, Oh JH. Outcomes of pelvic exenteration for recurrent or primary locally advanced colorectal cancer. Ann Surg Treat Res. 2015;89:131-7.

32. Quyn AJ, Austin KK, Young JM, Badgery-Parker T, Masya LM, Roberts R, et al. Outcomes of pelvic exenteration for locally advanced primary rectal cancer: overall survival and quality of life. Eur J Surg Oncol. 2016;42:823-8.

33. Vermaas M, Ferenschild FT, Verhoef C, Nuyttens JJ, Marinelli AW, Wiggers $T$, et al. Total pelvic exenteration for primary locally advanced andocally recurrent rectal cancer. Eur J Surg Oncol. 2007;33:452-8.

34. ke H, Shimada H, Yamaguchi S, Ichikawa Y, Fujii S, Ohki S. Outcome of total pelvic exenteration for primary rectal cancer, Dis Colon Rectum. 2003;46:474-80

35. Katory M, McLean R, Paez E, Kucukmetin A, Naik R. Short- and longterm outcomes following pelvic exenteration for gynae-oncological and colorectal cancers: a 9 year consecutive single-centre cohort study. Int J Surg. 2018:43:38-45.

36. Jimenez RE, Shoup M, Cohen AM, Paty PB, Guillem J, Wong WD. Contemporary outcomes of total pelvic exenteration in treatment of $\mathrm{co}$ lorectal cancer. Dis Colon Rectum. 2003;46:1619-25.

37. Krebs B, Kozelj M, Kavalar R, Gajzer B, Gadzijev EM. Prognostic value of additional pathological variables for long-term survival after curative resection of rectal cancer. World J Gastroenterol. 2006;12:4565-8.

38. Matsumoto K, Nakayama U, Inoue Y, Minagawa N, Katsuki T, Shibao K, et al. Lymphatic microvesseldensity is an independent prognostic factor in colorectal cancer. Dis Colon Rectum. 2007:50:308-14. 